\title{
Chondrule-Forming Shock Fronts in the Solar Nebula: A Possible Unified Scenario for Planet and Chondrite Formation
}

\author{
A. P. Boss ${ }^{1}$ and R. H. Durisen ${ }^{2}$ \\ ${ }^{1}$ Department of Terrestrial Magnetism, Carnegie Institution of Washington, 5241 Broad \\ Branch Road, NW, Washington, DC 20015-1305 \\ ${ }^{2}$ Department of Astronomy, Indiana University, 727 E. 3rd St., Bloomington, IN \\ 47405-7105 \\ E-mail: boss@dtm.ciw.edu (A.P.B.); durisen@astro.indiana.edu (R.H.D.)
}

\begin{abstract}
Chondrules are mm-sized spherules found throughout primitive, chondritic meteorites. Flash heating by a shock front is the leading explanation of their formation. However, identifying a mechanism for creating shock fronts inside the solar nebula has been difficult. In a gaseous disk capable of forming Jupiter, the disk must have been marginally gravitationally unstable at and beyond Jupiter's orbit. We show that this instability can drive inward spiral shock fronts with shock speeds of up to $\sim 10 \mathrm{~km} \mathrm{~s}^{-1}$ at asteroidal orbits, sufficient to account for chondrule formation. Mixing and transport of solids in such a disk, combined with the planet-forming tendencies of gravitational instabilities, results in a unified scenario linking chondrite production with gas giant planet formation.
\end{abstract}

Subject headings: solar system: formation - planetary systems - accretion, accretion disks

\section{Introduction}

Perhaps the most long-standing problem in all of meteoritics (Sorby 1877; Hewins 1996) is the search for an appropriate heat source for melting the chondrules that constitute the bulk of many primitive meteorites. Chondrules, cm-sized refractory inclusions, and micronand smaller-scale matrix particles came together to form the chondritic meteorites. These three components appear to have been the major solid constituents of the solar nebula, at least inside Jupiter's orbit. Their formation and assembly into chondrites provides strong constraints on processes occurring during the earliest phases of Solar System origin. 
The chondrule precursors are thought to have been aggregates of ferromagnesian silicate dust grains that were heated to their liquidus temperatures through multiple flash heating events, as witnessed by their textures, inferred cooling rates, occurrence of compound chondrules, and the occasional presence of fragments of relict chondrules inside other chondrules (Hewins 1996). Shock waves within the solar nebula are one possible means for accomplishing this thermal processing (Hood \& Horanyi 1991, 1993). Detailed studies have shown that such waves are able to melt suitable chondrule precursors provided that the shock waves move with respect to the precursor aggregates at speeds in the range of 6 to $9 \mathrm{~km} \mathrm{~s}^{-1}$ (Iida et al. 2001; Desch \& Connolly 2002; Ciesla \& Hood 2002).

In this paper we present the results of numerical hydrodynamical models that identify a likely source for shock heating of chondrule precursors. We then use these results to create a unified scenario for the thermal processing of chondrules and mixing of chondrules, refractory inclusions, and matrix grains to form the chondritic meteorites.

\section{Sources of Shock Fronts}

A suitable mechanism for producing shock fronts inside the solar nebula must be identified for the chondrule formation question to be considered answered. Four different mechanisms have been proposed as sources of such shock fronts. First, pre-existing dust grains would be slowed down and heated by frictional gas drag as they enter the solar nebula by passing through the accretion shock at the nebula's surface (Wood 1984; Ruzmaikina 1994). However, this process does not explain the presence of relict grains in chondrules, or the unlikely growth to mm-size (or larger, for fluffy aggregates) of grains in the relatively low density, earlier phases of presolar and interstellar cloud evolution. Second, the accretion of gas by the solar nebula may have been punctuated by the infall of clumps of gas and dust whose impact would have launched shock waves into the disk (Boss \& Graham 1993; Hood \& Kring 1996). Models have found that clumps need to have masses of $\sim 10^{27} \mathrm{~g}$ or more to produce the desired strong shock waves (Tanaka et al. 1998). Infalling clumps with masses greater than this have been inferred in the B335 molecular cloud (Velusamy, Kuiper, \& Langer 1995), but these clumps may be too distended to produce an impulsive, shockforming impact. Third, bow shocks driven by 1000-km-size planetesimals moving on highly eccentric orbits are another possibility (Hood 1998; Ciesla, Hood, \& Weidenschilling 2004). However, achieving such highly eccentric orbits in the asteroid region requires resonant gravitational interactions with a Jupiter-like planet (Weidenschilling, Marzari, \& Hood 1998). In the standard picture of giant planet formation by core accretion, Jupiter takes several million years (Ma) or more to form (Inaba, Wetherill, \& Ikoma 2003), whereas some chondrules 
were formed at essentially the same time as the earliest solids (the calcium, aluminum-rich inclusions, or CAIs) were formed (Bizzarro, Baker, \& Haack 2004).

The fourth mechanism is shock fronts associated with nonaxisymmetric disk structures such as spiral arms (Hood \& Horanyi 1991; Wood 1996). This possibility has been strongly supported by recent calculations of the evolution of gravitationally unstable disks (Boss 2002; Pickett et al. 2003). The solar nebula is likely to have been at least marginally gravitationally unstable during a portion of its evolution, because, regardless of mechanism, the formation of Jupiter requires the mass of the solar nebula to be $\sim 0.1 M_{\odot}$. Recent models of Jupiter formation by the core accretion mechanism require a disk with a mass of at least $0.08 M_{\odot}$ in order to form Jupiter within 3.8 Ma (Inaba et al. 2003). With outer disk temperatures of $\sim 28 \mathrm{~K}$ (Kawakita et al. 2001), such a disk would be marginally gravitationally unstable to the growth of density perturbations that would form strong spiral arms and possibly lead to the rapid formation of Jupiter (Boss 1997, 2002). The question then becomes whether a disk which is gravitationally unstable at and beyond Jupiter's 5.2 AU orbital distance is able to drive shock fronts into the asteroidal regions $(\sim 2.5 \mathrm{AU})$ where they could have been responsible for in situ thermal processing of solids.

\section{Numerical Methods}

We present here the results of a three dimensional, gravitational hydrodynamics calculation of the time evolution of a gravitationally unstable disk which directly addresses this question. The calculation includes a full treatment of disk thermodynamics and radiative transfer in the diffusion approximation. The numerical code solves the equations of hydrodynamics on a spherical coordinate $(r, \theta, \phi)$ grid with 112 radial grid points spread between $2 \mathrm{AU}$ and $20 \mathrm{AU}, 23 \theta$ grid points between the midplane and the rotation axis, concentrated around the midplane to provide adequate vertical spatial resolution, and 256 azimuthal grid points uniformly spaced in $\phi$. The calculation is identical to previous calculations (e.g., Boss 2002), except for being extended inward from 4 AU to 2 AU. The Poisson equation for the gravitational potential is solved by a spherical harmonic $\left(Y_{l m}\right)$ expansion including terms up to $l, m=32$. The disk has a mass of $0.096 M_{\odot}$ between $2 \mathrm{AU}$ and $20 \mathrm{AU}$. The initial outer disk temperature is $40 \mathrm{~K}$, leading to an initial value of the Toomre $Q$ stability parameter of $Q_{i} \sim 1.3$ beyond $5 \mathrm{AU}$. Studies of gravitational instabilities in disks generally show that disks are unstable to development of nonaxisymmetric structure for $Q_{i}<1.5$ to 1.7 (e.g., Boss 2002, Pickett et al. 2003). The inner regions have $Q_{i}>1.7$, indicating stability to density perturbations, because the initial disk has a radial temperature profile that rises to $1200 \mathrm{~K}$ at $2 \mathrm{AU}$. Hence any spiral structures that might form in the inner disk must be 
driven by gravitational forces from nonaxisymmetric structures in the outer disk.

\section{Results}

A common outcome of the evolution of gravitationally unstable disks is the formation of strong, transient shock fronts in the inner disk, driven by spiral arms and clumps at greater distances (Fig. 1). For one-armed ( $m=1$ modes) spiral arms of the type seen in Fig. 1, the inner Lindblad resonance occurs at angular velocity $\Omega=\infty$, or zero radius, so that a single clump can drive spiral waves right down to the protostar. The disk model exhibits this behavior, showing the presence of a strong shock front between 2 and 3 AU. A factor of 100 increase in midplane gas density occurs when passing through this shock front. This shock front is being driven by the gravitational forces associated with the clumps and spiral arms that have formed primarily between $5 \mathrm{AU}$ and $10 \mathrm{AU}$ in the disk. Note, however, that even if these clumps do not succeed in forming giant planets, their transient existence will still drive shock fronts at 2.5 AU, permitting thermal processing of chondrule precursors from essentially $t=0$ for the solar nebula, the time at which CAIs formed some 4,567 Ma ago (Amelin et al. 2002). In addition, once Jupiter forms by either core accretion or disk instability, it may continue to drive strong shock fronts in the inner disk similar to those seen in Fig. 1 for as long as the inner disk gas remains. Thus, chondrule formation is expected to proceed for several Ma, the estimated lifetime of the inner solar nebula.

The spiral pattern producing the inner shock fronts rotates at a speed governed roughly by the Keplerian angular velocity of the clumps orbiting beyond $5 \mathrm{AU}$. As a result, there can be a large difference in orbital velocity between this spiral pattern and dust aggregates moving with the gas on nearly Keplerian orbits at orbital radii inside (or outside) the distances of the clumps (Wood 1996). For a spiral pattern rotating with the angular velocity appropriate for a clump orbiting at $5.2 \mathrm{AU}$, the orbital velocity difference is $12 \mathrm{~km} \mathrm{~s}^{-1}$ between this spiral pattern at 2.5 AU and solids orbiting with the gas at 2.5 AU. However, it is only the component of this velocity difference perpendicular to the shock front that is relevant for thermal processing. Inner and outer shock fronts are usually so tightly wound that solids moving with the gas through such shocks would strike them at a low angle and hence with a velocity difference considerably less than $12 \mathrm{~km} \mathrm{~s}^{-1}$. At the time shown in Fig. 1, however, the inner shock is oriented nearly obliquely to the direction of motion of orbiting solids, at an angle of $\sim 60$ degrees, leading to a shock speed of $\sim 10 \mathrm{~km} \mathrm{~s}^{-1}$, more than sufficient for melting chondrule precursor dust aggregates. This transient shock structure is the driving motivation for this paper. Most of the time the angle around 2.5 AU is closer to $\sim 30$ degrees or smaller, leading to a shock speed of $\sim 6 \mathrm{~km} \mathrm{~s}^{-1}$ or less. High-angle shocks have not been 
seen in the outer disk in this model.

Multiple chondrule heating events may be required to produce porphyritic texures in chondrules (Hewins \& Fox 2004), and transient though persistent spiral waves lasting for several Ma are clearly able to satisfy that constraint. However, the number of heating events may also be limited by the need to preserve chondrule rims (Hewins 1996). Global simulations of unstable disks integrated long enough for the gravitational instabilities to settle into an asymptotic turbulent behavior (Mejía et al. 2005), as well as the model presented in Fig. 1, show, however, that shock fronts with the required conditions (shock speed, pre- and postshock densities) are by no means a permanent feature. Rather, shocks which appear to have roughly the desired characteristics are transients in a chaotic environment. Moreover, it is possible that gravitational instabilities in the solar nebula were intermittent due to the episodic build up of mass in a "dead zone" where radial transport by magnetohydrodynamic turbulence was inhibited (Gammie 1996; Armitage, Livio, \& Pringle 2001). Episodes of rapid transport by gravitational instabilities may have been associated with FU Orionis outbursts. Future work is needed to estimate how often a protoplanetary disk is likely to produce a shock front suitable for chondrule formation.

\section{Unified Scenario for Chondrules and CAIs}

In Fig. 2 we present a unified scenario for the chronology of the early Solar System and the thermal processing of solids. Models of gravitationally unstable disks have shown (Boss 2004) that mixing and transport of mm-sized and even $\mathrm{cm}$-sized solids occurs on a short time scale $(\sim 0.001 \mathrm{Ma})$, faster than such solids could spiral in to the protosun by gas drag and be lost. In addition, in a marginally gravitationally unstable nebula of the type needed to form Jupiter by either core accretion or disk instability, the nebula will have transient spiral arms and rings that produce local pressure maxima in the disk midplane. Because of these midplane pressure maxima, solids will feel headwinds and tailwinds that drive them toward the centers of the arms and rings (Haghighipour \& Boss 2003; Rice et al. 2004; Durisen et al. 2005). This prevents the loss of solids by migration toward the protosun that would otherwise result from gas drag due to headwinds in a nebula where the gas pressure decreased monotonically with distance from the protosun. Instead, the CAIs, chondrules, and smaller dust grains will remain tied to the gas of the gravitationally evolving disk, and concentration of solids in dense structures will accelerate formation of planetesimals, planetary embryos, and asteroidal parent bodies through mutual collisions. These bodies are large enough to be effectively decoupled from the disk gas, and so remain behind after the inner disk gas is depleted. In the magnetically "dead zone" model of solar 
nebula evolution, chondrite parent-body forming events may occur episodically. Within 3 $\mathrm{Ma}$, the inner disk is largely cleared of gas and primordial dust, and over the next $\sim 30$ Ma the terrestrial planets then grow by collisional accumulation of the planetesimals and planetary embryos previously formed there.

\section{Conclusions}

Spiral waves driven by gravitational instabilities appear to be a natural, almost unavoidable means for thermally processing chondrule precursors in the early solar nebula. Chondrules that were formed in the asteroidal region of the solar nebula would have been mixed together with CAIs that formed closer to the protosun and with smaller, unheated grains (matrix) to form the chondritic meteorites. The mixing, segregration by dense structures, and thermal processing of chondritic material may thus be intimately connected with dynamic processes in the gas disk that led to gas giant planet formation in the outer nebula.

We thank A. C. Boley and the referee for useful comments. A.P.B.'s research is supported in part by the NASA Planetary Geology and Geophysics Program under grant NNG04GG15G and the NASA Origins of Solar Systems Program under grant NAG5-11569. Calculations were performed on the Carnegie Alpha Cluster, the purchase of which was supported in part by NSF MRI grant AST-9976645. R.H.D.'s research was supported in part by the NASA Origins of Solar Systems Program grant NAG5-11964.

\section{REFERENCES}

Amelin, Y., Krot, A. N., Hutcheon, I. D., \& Ulyanov, A. A. 2002, Science, 297, 1678

Armitage, P. J., Livio, M., \& Pringle, J. E. 2001, MNRAS, 324, 705

Bizzarro, M., Baker, J. A., \& Haack H. 2004, Nature, 431, 275

Boss, A. P. 1997, Science, 276, 1836

—. 2002, ApJ, 576, 462

- 2004, ApJ, 616, 1265

Boss, A. P., \& Graham, J. A. 1993, Icarus, 106, 168

Ciesla, F. J., \& Hood, L. L. 2002, Icarus, 158, 281

Ciesla, F. J., Hood, L. L., \& Weidenschilling, S. J. 2004, Meteor. Planet. Sci., 39, 1809

Desch, S. J., \& Connolly, H. C., Jr. 2002, Meteor. Planet. Sci., 37, 183 
Durisen, R. H., Cai, K., Mejía, A. C., \& Pickett, M. K. 2005, Icarus, in press

Gammie, C. F. 1996, ApJ, 553, 174

Haghighipour, N., \& Boss, A. P. 2003, ApJ, 598, 1301

Hewins, R. H. 1996, in Chondrules and the Protoplanetary Disk, ed. R. H. Hewins, R. H. Jones, \& E. R. D. Scott (Cambridge: Cambridge Univ. Press), 3

Hewins, R. H., \& Fox, G. E. 2004, Geochim. Cosmochim. Acta, 68, 917

Hood, L. L. 1998, Meteor. Planet. Sci., 33, 97

Hood, L. L., \& Horanyi, M. 1991, Icarus, 93, 259

—. 1993, Icarus, 106, 179

Hood, L. L., \& Kring, D. A. 1996, in Chondrules and the Protoplanetary Disk, ed. R. H. Hewins, R. H. Jones, \& E. R. D. Scott (Cambridge: Cambridge Univ. Press), 265

Iida, A., Nakamoto, T., Susa, H., \& Nakagawa, Y. 2001, Icarus, 153, 430

Inaba, S. , Wetherill, G. W., \& Ikoma, M. 2003, Icarus, 166, 46

Kawakita, H. et al. 2001, Science, 294, 1089

Mejía, A. C., Durisen, R. H., Pickett, M. K., \& Cai, K. 2005, ApJ, in press

Pickett, B. K., Mejía, A. C., Durisen, R. H., Cassen, P. M., Berry, D. K., \& Link, R. P. 2003, ApJ, 590, 1060

Rice, W. K. M., Lodato, G., Pringle. J. E., Armitage, P. J., \& Bonnell, I. A. 2004, MNRAS, 355,543

Ruzmaikina, T. V., \& Ip, W. H. 1994, Icarus, 112, 430

Shu, F. H., Shang, H., Gounelle, M., Glassgold, A. E., \& Lee, T. 2001, ApJ, 548, 1029

Sorby, H. C. 1877, Nature, 15, 495

Tanaka, K. K., Tanaka, H., Nakazawa, K., \& Nakagawa, Y. 1998, Icarus, 134, 137

Velusamy, T., Kuiper, T. B. H., \& Langer, W. D. 1995, ApJ, 451, L75

Weidenschilling, S. J., Marzari, F., \& Hood, L. L. 1998, Science, 279, 681

Wood, J. A. 1984, Earth Planet. Sci. Lett., 70, 11

—. 1996, Meteor. Planet. Sci., 31, 641 


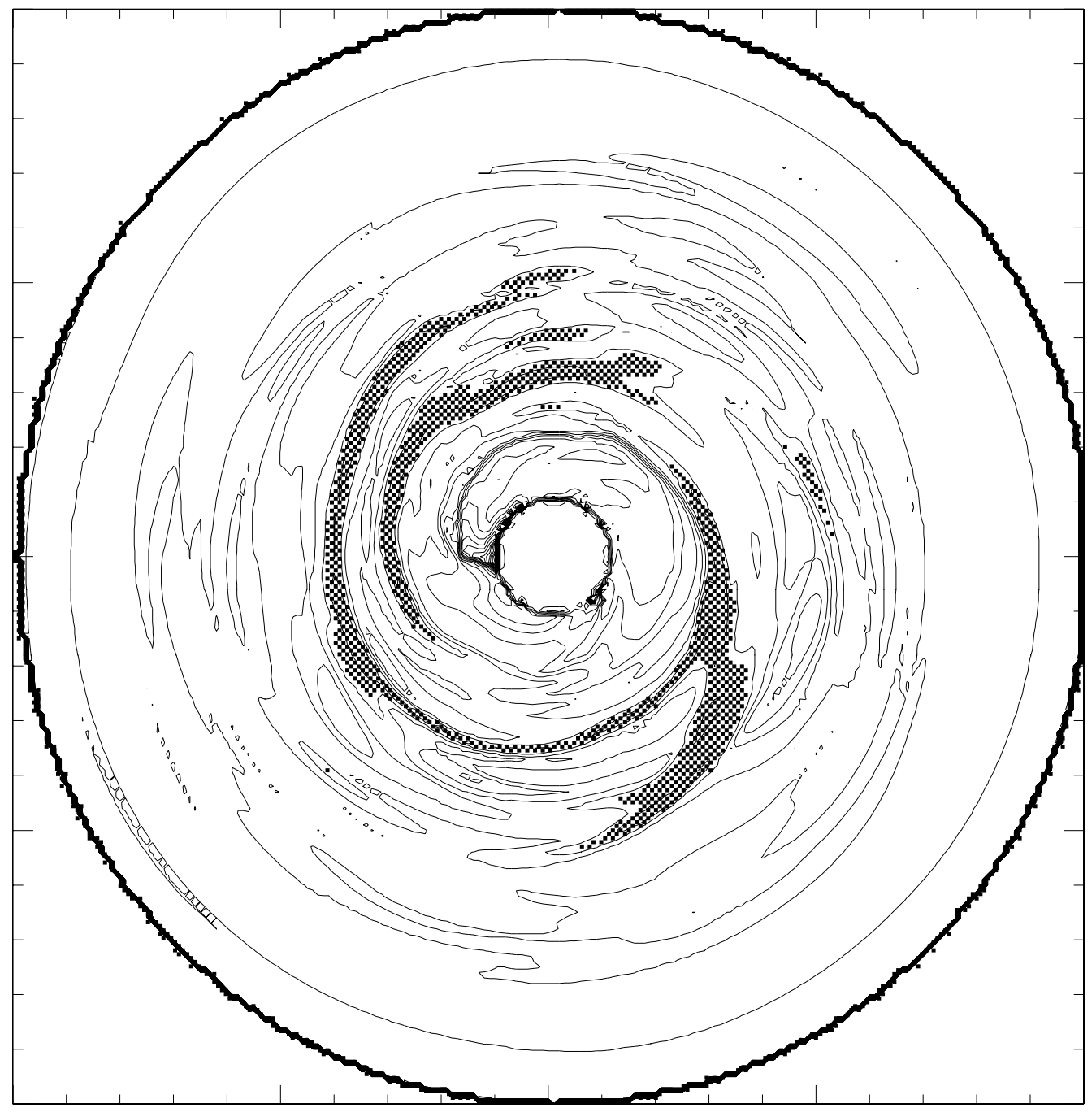

Fig. 1.- Density contours in the midplane of a gravitationally unstable disk after 252 years of evolution from an initially nearly axisymmetric state, showing a strong transient shock front located at 9 o'clock, just outside the inner boundary of radius 2 AU. Radius of the entire region shown is $20 \mathrm{AU}$. Cross-hatched regions denote regions with densities above $10^{-10} \mathrm{~g} \mathrm{~cm}^{-3}$. A solar-mass protostar lies at the center of the disk. Solids rotating in the counterclockwise direction between 2 and $3 \mathrm{AU}$ encounter the shock front at a speed of $\sim 10$ $\mathrm{km} \mathrm{s}^{-1}$. 
Fig. 2.- A unified scenario for the evolution of solids in the inner solar nebula. (a) At $t=0$ Ma, defined by the 4,567 Ma age of CAIs, CAIs are present in the disk, formed close to the protosun and possibly lofted by the protosun's bipolar outflow (Shu et al. 2001) to greater distances (streamlines above and below disk). The bulk of the disk is magnetically dead because of the low ionization fraction, while the surface of the disk is ionized and magnetically active (Gammie 1996). (b) The disk is marginally gravitationally unstable, resulting in the rapid inward and outward transport of CAIs and dust grains (Boss 2004). (c) Spiral arms form Jupiter-mass clumps as disk mixing and transport of solids continues. (d) The spiral arms and clumps at 5 AU and beyond drive strong shock fronts in the inner disk, capable of thermally processing precursor dust aggregates into chondrules. (e) Jupiter and Saturn form either rapidly (Boss 1997, 2001) or slowly (Inaba et al. 2003) but in either case continue to drive shock fronts intermittently at asteroidal distances. Chondrules, CAIs, and matrix-sized dust grains collide and form planetesimals and planetary embryos in the inner disk. (f) Within $3 \mathrm{Ma}$, the inner solar nebula is accreted by the protosun, leaving behind the rocky bodies that will collide over the next $\sim 30 \mathrm{Ma}$ to form the terrestrial planets and the asteroid belt. 


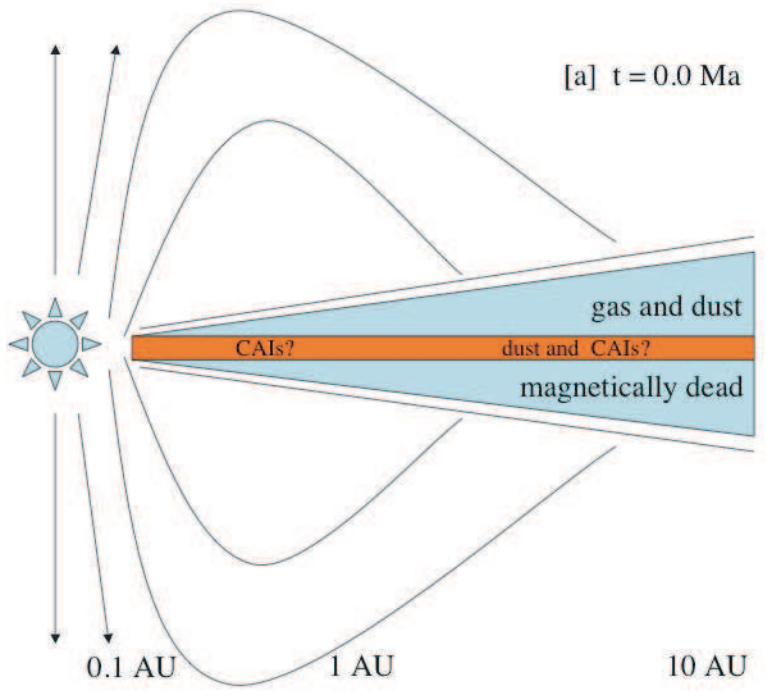

$-1 C$
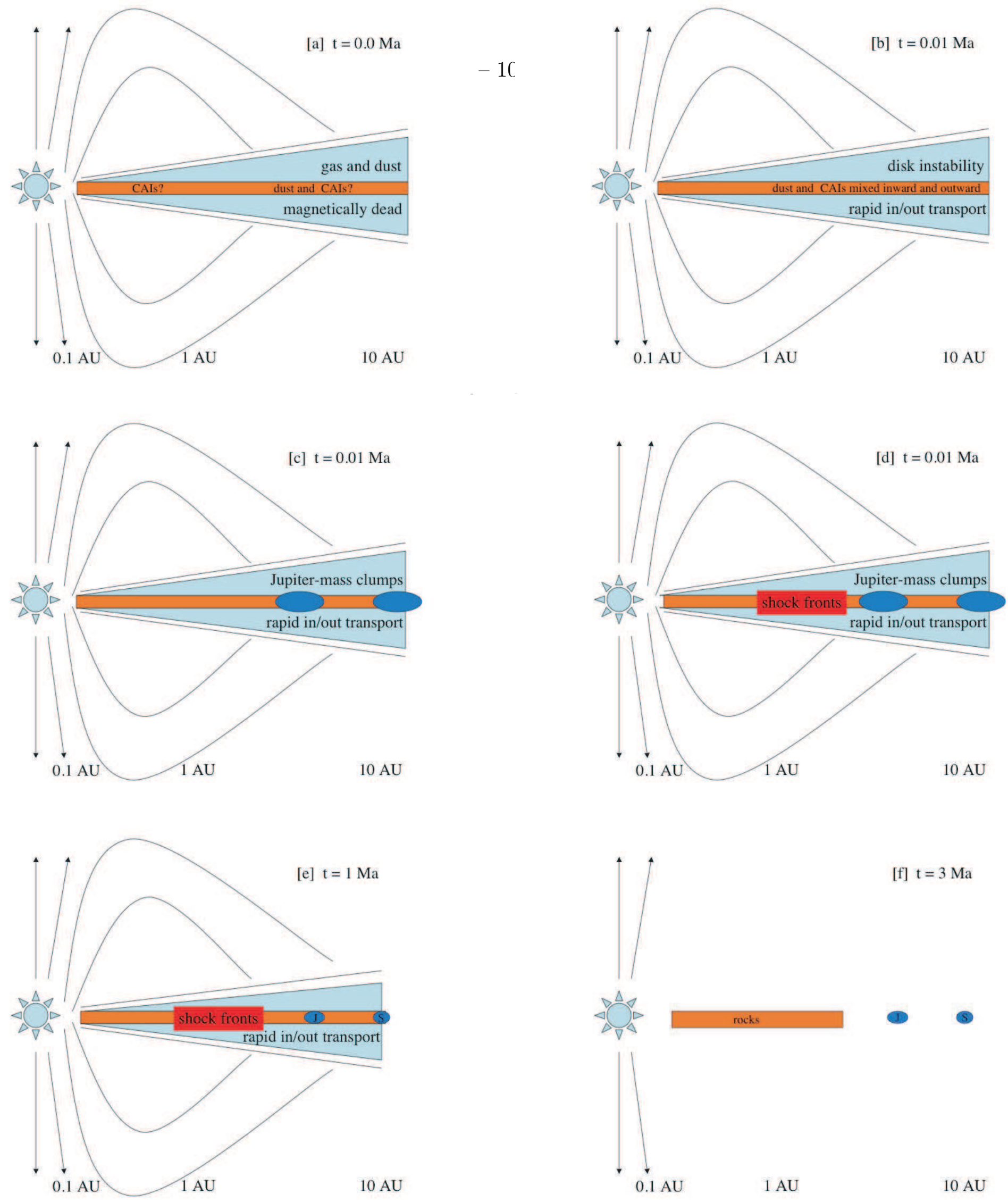